\title{
THE EFFECT OF TREATMENT ON CYTOKINE LEVELS IN NON MUSCLE INVASIVE BLADDER CANCER
}

\author{
Oktay Ucer ${ }^{1}$, Gokhan Temeltas ${ }^{2}$, Talha Muezzinoglu² ${ }^{2}$ Zeki Ari², and Funda Kosova ${ }^{2}$ \\ ${ }^{1}$ Affiliation not available \\ ${ }^{2}$ Celal Bayar University
}

November 29, 2020

\begin{abstract}
Objectives The aim of the present study was to investigate the effect of treatment on IL-1, IL-6, IL- 8 and neopterin levels in patients with non-muscle invasive bladder cancer (NMIBC). Methods Thirty patients with NMIBC and 30 age-matched controls were included in the study. Preoperative, postoperative first control (at two weeks after second transurethral resection of bladder tumor (TURBT)) and second control (at the end of intravesical immunotherapy) blood samples were analyzed by ELISA to determine IL-1, IL-6, IL-8 and neopterin levels. The mean cytokine levels of the patients were statistically compared as well as comparing the patients' and controls' levels. Results There were no statistically significant differences between the mean IL-1, IL-6, IL-8 and neopterin levels of the patient and control group before initial TURBT. In the patient group, there were no statistically significant differences in the IL- 6 and IL- 8 levels after both TURBT and intravesical BCG therapy. The mean of preoperative IL-1 and neopterin levels significantly decrease after TURBT $(\mathrm{p}<0.05=)$. However this reduction do not continue after intravesical BCG instillations. Conclusions The finding of this study showed that the IL-1, IL-6, IL- 8 and neopterin levels of the patients with NMIBC were similar to the levels of healthy controls. The IL-1 and neopterin levels significantly decreased after TURBT. But this reductions did not continue after intravesical BCG instillation. These findings demonstrate that IL-1 and neopterin levels decrease after TURBT due to the reduction of tumor weight or tumor removal.
\end{abstract}

\section{THE EFFECT OF TREATMENT ON CYTOKINE LEVELS IN NON MUSCLE INVASIVE BLADDER CANCER}

\section{Abstract \\ Objectives}

The aim of the present study was to investigate the effect of treatment on IL-1, IL- 6 , IL- 8 and neopterin levels in patients with non-muscle invasive bladder cancer (NMIBC).

\section{Methods}

Thirty patients with NMIBC and 30 age-matched controls were included in the study. Preoperative, postoperative first control (at two weeks after second transurethral resection of bladder tumor (TURBT)) and second control (at the end of intravesical immunotherapy) blood samples were analyzed by ELISA to determine IL-1, IL-6, IL-8 and neopterin levels. The mean cytokine levels of the patients were statistically compared as well as comparing the patients' and controls' levels.

\section{Results}

There were no statistically significant differences between the mean IL-1, IL-6, IL-8 and neopterin levels of the patient and control group before initial TURBT. In the patient group, there were no statistically significant differences in the IL-6 and IL- 8 levels after both TURBT and intravesical BCG therapy. The 
mean of preoperative IL- 1 and neopterin levels significantly decrease after TURBT $(\mathrm{p}<0.05=)$. However this reduction do not continue after intravesical BCG instillations.

\section{Conclusions}

The finding of this study showed that the IL-1, IL-6, IL-8 and neopterin levels of the patients with NMIBC were similar to the levels of healthy controls. The IL-1 and neopterin levels significantly decreased after TURBT. But this reductions did not continue after intravesical BCG instillation. These findings demonstrate that IL-1 and neopterin levels decrease after TURBT due to the reduction of tumor weight or tumor removal.

\section{What is already known about this topic?}

Proinflammatory cytokines including IL -1, IL-6, IL-8 and neopterin play an important role in the active immune response in cancer. However we do not know what is happening in these cytokines levels after treatment of cancer (surgical resection and medical therapy).

\section{What does this article add?}

Some cytokines such as the IL-1 and neopterin levels decrease after surgical resection of tumor in non-muscle invasive bladder cancer due to the decrease in tumor weight or tumor removal.

\section{Introduction}

Bladder cancer is the seventh most commonly diagnosed cancer in men, in whom it is approximately four times more common than in women. At the time of diagnosis, approximately $75 \%$ of patients present with non-muscle-invasive bladder cancer (NMIBC), which is confined to the mucosa (Ta, carcinoma in situ) or submucosa (T1)(1). Many meta-analyses have confirmed that intravesical bacillus Calmette-Guerin (BCG) immunotherapy after transurethral resection of bladder tumor (TURBT) is superior to TURBT alone for preventing the recurrence and/or progression of NMIBC (2-5). Therefore, European Association of Urology (EAU) Guidelines on NMIBC recommend intravesical BCG immunotherapy after TURBT in patients with intermediate- or high-risk NMIBC (1).

Some studies have investigated the effect of intravesical BCG immunotherapy on some angiogenetic factors and cytokines such as interleukin (IL)-8 in patients with NMIBC $(6,7)$. They suggested that these factors and cytokines might be used for the follow up after intravesical BCG immunotherapy in NMIBC. The antitumor features of intravesical BCG immunotherapy primarily depend on the BCG-induced inflammatory response (8) that is impaired in NMIBC as reflected by an imbalanced production of immuno-modulating cytokines (9). It is important to more fully understand the significance of these cytokines for predicting outcome of intravesical BCG immunotherapy in NMIBC. Some studies have showed that proinflammatory cytokines including IL -1, IL-6, IL-8 and neopterin played an important role in the active immune response in cancer (10-12). Therefore, these cytokines levels may decrease after treatment of cancer (surgical resection and medical therapy). The aim of the present study was to compare IL-1, IL-6, IL-8 and neopterin levels of the patients with NMIBC and healthy controls, and also investigate the changes in these cytokines and neopterin levels after TURBT and intravesical BCG immunotherapy.

\section{Material and Methods}

\section{Study Population}

A total of 41 patients with newly diagnosed bladder cancer who underwent initial TURBT and 30 agematched controls were enrolled in the study. Inclusion criteria were newly diagnosed intermediate or high risk NMIBC who received an second TURBT and 6 doses of BCG. Exclusion criteria were low risk NMIBC, T2 and BCG toxicity. 3 patients with low-risk NMIBC and 3 patients with MIBC (T2) after initial TURBT were excluded from the study. 35 patients underwent second TURBT at 4-6 weeks after initial TURBT. 3 patients with MIBC after second TURBT were excluded from the study. 32 patients were received intravesical BCG therapy once a week for 6 weeks. 2 patients were excluded from the study because of BCG toxicity. The patients' enrolment algorithm has been illustrated in Figure 1. The study group comprised 30 patients 
with intermediate- or high-risk NMIBC and 30 age-matched controls. IL-1, IL-6, IL-8 and neopterin levels were measured by enzyme-linked immunosorbent assay (ELISA) in the blood samples of all the patients before initial TURBT (preoperative group), at 2 weeks after second TURBT (postoperative first control group) and at 2 weeks after the end of induction intravesical BCG immunotherapy (postoperative second control group). These cytokines were also measured by ELISA in the blood samples of controls. Informed consent was obtained from all the patients and controls who participated in the study and the Local Ethics Committee approved the study protocol.

Statistical analysis

Statistical analysis was performed using "Statistical Package for Social Sciences 22.0 software (SPSS 22.0 for MAC)". Descriptive statistics were presented as mean \pm standard deviation, frequency and percentages. Shapiro-Wilk test was used to determine whether the data showed normal distribution. It was observed that all the parameters we examined conformed to normal distribution. Student's t-test ( $\mathrm{T}$ test in independent groups) was used to compare normally distributed continuous variables between the control and patient groups before initial TURBT. Mann-Whitney U test was used to compare not normally distributed continuous variables between the control and patient groups before initial TURBT. The comparison between the patient and control groups for sex was performed using the chi-square test. In the patient group, repeated measures analysis of variance (ANOVA) was used to compare variables between the preoperative, postoperative first control and postoperative second control. When appropriate, a Bonferroni t-test was used as a post hoc test. $\mathrm{P}<0.05$ was defined as the statistical significance level.

\section{Results}

The mean age of the patient (22 males and 8 females) and control (21 males and 9 females) groups were 57.3 \pm 7.8 and $55.8 \pm 9.0$ years, respectively $(\mathrm{p}=0.33)$. There were no statistically significant differences between the mean IL-1, IL-6, IL-8 and neopterin levels of the patient and control group before initial TURBT (Table 1 ). In the patient group, there were no statistically significant differences in the preoperative IL- 6 and IL8 levels after both TURBT and intravesical BCG therapy (respectively, $X^{2}(2)=1.333, \mathrm{p}=0.513$ and $X^{2}$ $(2)=2.778, \mathrm{p}=0.249$ ). The mean IL-1 levels of the preoperative, postoperative first control and postoperative second control were $4.09 \pm 2.43,3.99 \pm 2.49$ and $3.95 \pm 2.30$, respectively $\left(X^{2}(2)=10.500, \mathrm{p}=0.005\right)$. In the pairwise comparisons analysis, there was a significant decrease between the IL-1 levels in the preoperative and postoperative first control $(\mathrm{p}<0.05)$ (Figure 2). The mean neopterin levels in the preoperative, postoperative first control and postoperative second control were $3.31 \pm 1.18,2.89 \pm 1.60$ and $2.89 \pm 1.35$, respectively $\left(X^{2}\right.$ $(2)=14.941 \mathrm{p}=0.001)$. In the pairwise comparisons analysis, there were decreases between neopterin levels in the preoperative and postoperative first control $(\mathrm{p}<0.05)$, in the preoperative and postoperative second controls $(\mathrm{p}<0.05)$ (Figure 3).

\section{Discussion}

This study demonstrated that the IL-1 and neopterin levels of the patients with NMIBC significantly decreased after TURBT, however these decreases did not continue after intravesical BCG immunotherapy (Figure 2 and 3). Although the IL-1 levels of the patients were higher than the levels of the controls, this difference was not statistically significant. According to our best knowledge, there has been no study in literature to investigate IL-1 levels in blood samples of patients with NMIBC. Some studies have evaluated IL-1 levels in urine samples of patients with NMIBC in hours after intravesical BCG therapy $(13,14)$. They found that the urine IL-1 levels increased after intravesical BCG therapy and reported that the result reflected the local inflammatory response to BCG. They also suggested further studies that would evaluate the possible role of IL-1 against NMIBC(14). We investigated the effect of treatment (TURBT and intravesical BCG) on the IL-1 level in the blood of the patients with NMIBC and our results showed that TURBT caused a significant decrease in the IL-1 level. This finding is novel to the literature. This reduction may be related to the decrease in tumor weight or tumor removal.

Similar to the reduction of the IL-1 level, the neopterin levels of the patients with NMIBC decreased after TURBT in our study. According to the best of our knowledge, there is only one study that investigated 
neopterin levels in blood samples of patients with NMIBC (15). In this study, the authors measured the neopterin levels before, at $4^{\text {th }}, 24^{\text {th }}, 48^{\text {th }}$ and $96^{\text {th }}$ hour after intravesical BCG and investigated the role in immune response of neopterin after BCG. They found that the highest blood neopterin levels were found 48 hours after intravesical BCG therapy and were significantly higher than levels before BCG, 4 hours and 24 hours after BCG. They also suggested that neopterin in serum might be used as a parameter for monitoring treatment course. We differently investigated neopterin levels after TURBT. Similar to the decrease of IL-1, the reduction of neopterin levels after TURBT may be related to the decrease in tumor weight and tumor removal.

There have been some studies that investigated IL-6 levels in patients with bladder cancer (16-20). Only one (20) of these studies evaluated this cytokine in blood samples of patients. In the other studies, it was measured either in urine samples $(18,19)$ or tumor issues $(16,17)$. Kumari et al $(20)$ evaluated serum the IL-6 levels in 72 patients with bladder cancer (52 NMIBC and $20 \mathrm{MIBC}$ ). They divided the patients into 2 groups according to the presence of recurrence and found that the IL-6 levels of the patients with recurrent were higher than the patients with non-recurrent. They also reported the association high concentration of some cytokines such as IL-6 with poor recurrence free survival in the patients with bladder cancer. However, in their study, the IL-6 levels were not compared before and after the treatment of bladder cancer. Therefore we do not know the change of IL-6 levels after the treatment in their study. We differently investigated the IL-6 levels before and after TURBT and intravesical BCG instillation in only NMIBC. The results of our study showed that the IL-6 levels before the treatment did not statistically change after both TURBT and intravesical BCG therapy. Therefore, according to our outcomes, IL-6 is not a proper biomarker to follow patients with NMIBC.

Similar to IL-6, there have been some studies that investigated IL-8 levels in urine of patients with NMIBC $(19,20)$. The results of these studies showed that there was a significant relationship between high IL-8 levels and poor prognosis in the follow-up of NMIBC. However, they did not investigate the IL-8 levels in the blood of the patients. We compared the IL- 8 levels in the blood of the patients with NMIBC with the healthy controls and found that there was no significant difference. We also compared the preoperative IL-8 levels with the IL-8 levels after TURBT and intravesical BCG therapy. We found that the preoperative IL- 8 levels did not change after treatment of NMIBC. Therefore, although the previous studies suggested that urinary IL-8 levels in patients might be used to predict the prognosis of NMIBC, the finding of our study showed that serum IL-8 level is not an available cytokine to use in patients with NMIBC.

There were some limitations in our study. The first one was that we did not follow the patients after intravesical BCG therapy. Therefore we could not assess the progression and recurrence status of the patients. The other limitations were small sample size and choice of cut-off times for blood sampling. The last limitation was that we measured the cytokines only in blood samples and did not perform urine tests.

\section{Conclusion}

The finding of this study showed that IL-1, IL-6, IL- 8 and neopterin levels in blood of patients with NMIBC were similar to the levels of healthy controls. Although the IL-6 and IL-8 levels did not change after TURBT and intravesical BCG instillation, the IL-1 and neopterin levels significantly decreased after TURBT. But this reductions in the IL-1 and neopterin levels did not continue after intravesical BCG instillation. In conclusion, our findings demonstrated that the IL-1 and neopterin levels decrease after TURBT due to the decrease in tumor weight or tumor removal. We suggest further studies that will investigate IL-1 and neopterin in long term follow-up after TURBT.

\section{Acknowledgement}

This work was supported by Research Fund of Manisa Celal Bayar Univeristy.

\section{Ethical standards}

The Local Ethics Committee approved the study protocol. 


\section{Conflict of interest}

There are no conflicts of interest to be stated for the corresponding author and all co-authors.

\section{References}

1. Babjuk M, Burger M, Compérat EM, Gontero P, Mostafid AH, Palou J, et al. European Association of Urology Guidelines on Non-muscle-invasive Bladder Cancer (TaT1 and Carcinoma In Situ) - 2019 Update. Eur Urol 2019; 76(5): 639-657.

2. Malmström PU, Sylvester RJ, Crawford DE, Friedrich M, Krege S, Rintala E, et al. An individual patient data meta-analysis of the long-term outcome of randomised studies comparing intravesical mitomycin C versus bacillus Calmette-Guérin for non-muscle-invasive bladder cancer. Eur Urol 2009; 56(2): 247-56.

3. Han RF, Pan JG. Can intravesical bacillus Calmette-Guérin reduce recurrence in patients with superficial bladder cancer? A meta-analysis of randomized trials. Urology 2006; 67(6): 1216-23.

4. Böhle A, Bock PR. Intravesical bacille Calmette-Guérin versus mitomycin C in superficial bladder cancer: formal meta-analysis of comparative studies on tumor progression. Urology 2004; 63(4): 682-6

5. Sylvester RJ, van der MEIJDEN AP, Lamm DL. Intravesical bacillus Calmette-Guerin reduces the risk of progression in patients with superficial bladder cancer: a meta-analysis of the published results of randomized clinical trials. J Urol 2002; 168(5): 1964-70.

6. Temeltas G, Kosova F, Ucer O, Muezzinoglu T, Ari Z. Effects of Treatment on Angiogenic (Vascular Endothelial Growth Factor-2 and Matrix Metalloproteinase-2) and Antiangiogenic (Endostatin and Thrombospondin-1) Factors in Non-muscle Invasive Bladder Carcinoma. J of Urol Surg 2017; 4: 71-75.

7. Qu K, Gu J, Ye Y, Williams SB, Dinney CP, Wu X, Kamat A. High baseline levels of interleukin8 in leukocytes and urine predict tumor recurrence in non-muscle invasive bladder cancer patients receiving bacillus Calmette-Guerin therapy: A long-term survival analysis. Oncoimmunology 2017; 6(2): e1265719.

8. Bohle A, Brandau S. Immune mechanisms in bacillus Calmette-Guerin immunotherapy for superficial bladder cancer. J Urol 2003; 170: 964-9

9. Takeuchi A, Dejima T, Yamada H, Shibata K, Nakamura R, Eto M, et al. IL-17 production by gammadelta $\mathrm{T}$ cells is important for the antitumor effect of Mycobacterium bovis bacillus Calmette-Guerin treatment against bladder cancer. Eur J Immunol 2011; 41: 246-51.

10. Ma Y, Ren Y, Dai ZJ, Wu CJ, Ji YH, Xu J. IL-6, IL-8 and TNF- $\alpha$ levels correlate with disease stage in breast cancer patients. Adv Clin Exp Med 2017; 26(3): 421-426.

11. Mantovani A, Barajon I, Garlanda C. IL-1 and IL-1 regulatory pathways in cancer progression and therapy. Immunol Rev 2018; 281(1): 57-61.

12. Isci Bostanci E, Ugras Dikmen A, Girgin G, Gungor T, Baydar T, Nuri Danisman A. A New Diagnostic and Prognostic Marker in Endometrial Cancer: Neopterin. Int J Gynecol Cancer 2017; 27(4): 754-758.

13. $\left.{ }^{* *}\right)$ De Boer EC, De Jong WH, Steerenberg PA, Aarden LA, Tetteroo E, De Groot ER, et al. Induction of urinary interleukin-1 (IL-1), IL-2, IL-6, and tumour necrosis factor during intravesical immunotherapy with bacillus Calmette-Guérin in superficial bladder cancer. Cancer Immunol Immunother 1992; 34(5): 306-12.

14. (**)Böhle A, Nowc C, Ulmer AJ, Musehold J, Gerdes J, Hofstetter AG, et al. Detection of urinary TNF, IL 1, and IL 2 after local BCG immunotherapy for bladder carcinoma. Cytokine 1990; 2(3): $175-81$.

15. Mack D, Gomahr A, Herold M, Frick J. Neopterin as a monitoring parameter for treatment with BCG in superficial bladder cancer. Eur J Cancer 1995; 31A(6): 1025-6.

16. (*)Hwang EC, Jung SI, Lee HJ, Lee JJ, Kwon DD. Generation of potent cytotoxic T lymphocytes against in male patients with non-muscle invasive bladder cancer by dendritic cells loaded with dying T24 bladder cancer cells. Int Braz J Urol 2017; 43(4): 615-627.

17. (*)Chen MF, Lin PY, Wu CF, Chen WC, Wu CT. IL-6 expression regulates tumorigenicity and correlates with prognosis in bladder cancer. PLoS One 2013; 8(4): e61901.

18. (**)Cai T, Nesi G, Mazzoli S, Meacci F, Tinacci G, Luciani LG, et al. Prediction of response to bacillus 
Calmette-Guérin treatment in non-muscle invasive bladder cancer patients through interleukin-6 and interleukin-10 ratio. Exp Ther Med 2012; 4(3): 459-464.

19. Kamat AM, Briggman J, Urbauer DL, Svatek R, Nogueras González GM, Anderson R, et al. Cytokine Panel for Response to Intravesical Therapy (CyPRIT): Nomogram of Changes in Urinary Cytokine Levels Predicts Patient Response to Bacillus Calmette-Guérin. Eur Urol. 2016; 69(2): 197-200.

20. Kumari N, Agrawal U, Mishra AK, Kumar A, Vasudeva P, Mohanty NK, et al. Predictive role of serum and urinary cytokines in invasion and recurrence of bladder cancer. Tumour Biol 2017; 39(4): 1-14.

Table 1. Mean IL-1, IL-6, IL-8 and neopterin levels of patient and control groups.

\begin{tabular}{llll}
\hline & $\begin{array}{l}\text { Patient group } \\
\text { (preoperative) Mean } \\
\pm \text { SD }\end{array}$ & $\begin{array}{l}\text { Control group Mean } \\
\pm \text { SD }\end{array}$ & P value \\
\hline IL-1 (pg/L) & $4.09 \pm 2.43$ & $3.65 \pm 1.30$ & 0.96 \\
IL-6 (ng/L) & $4.13 \pm 2.13$ & $3.82 \pm 1.24$ & 0.58 \\
IL-8 (ng/L) & $5.37 \pm 2.81$ & $4.32 \pm 1.50$ & 0.05 \\
Neopterin (nmol/L) & $3.31 \pm 1.21$ & $3.70 \pm 1.31$ & 0.34 \\
\hline
\end{tabular}

$\mathrm{P}<0.05$ is defined a statistically significant.

Figure 1. Flow diagram of the study participants.

TURBT: Transurethral resection of bladder tumor, NMIBC: Non-muscle-invasive bladder cancer, MIBC: Muscle-invasive bladder cancer, BCG: Bacillus Calmette-Guerin

Figure 2. The maen IL-1 levels of the patients in preoperative, postoperative first control and postoperative second control.

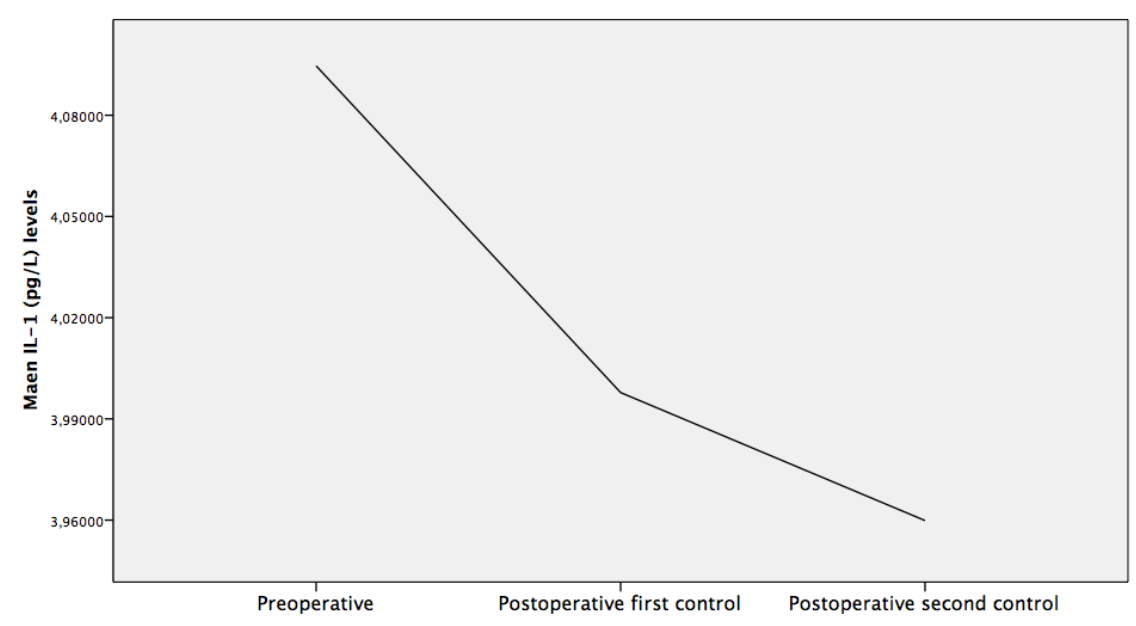




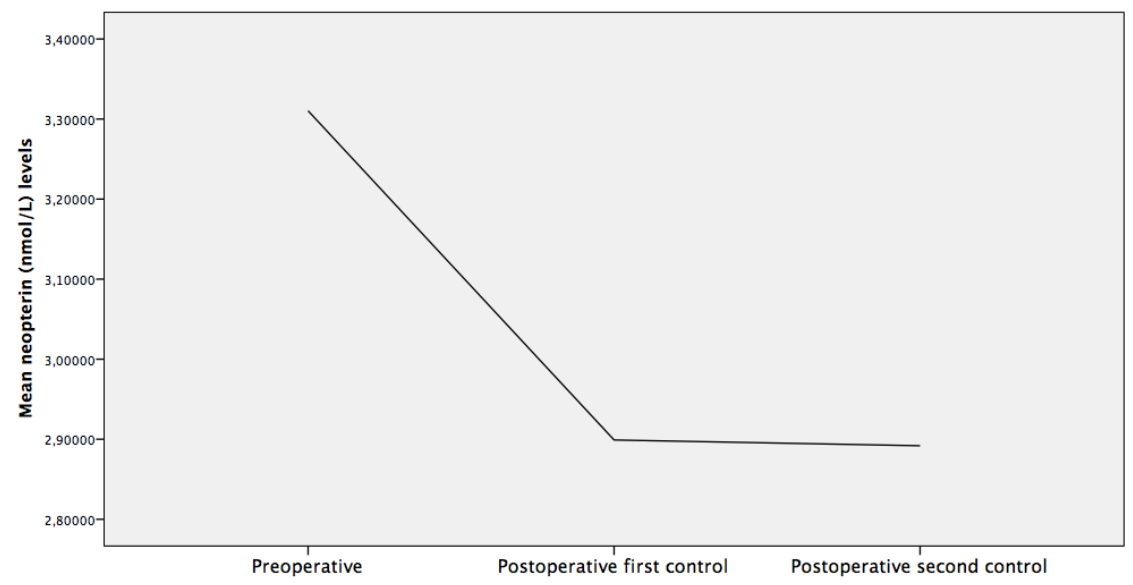

Figure 3. The mean neopterin levels of the patients in preoperative, postoperative first control and postoperative second control.

\section{Hosted file}

Table 1.pdf available at https://authorea.com/users/353510/articles/495949-the-effect-oftreatment-on-cytokine-levels-in-non-muscle-invasive-bladder-cancer

\section{Hosted file}

Figure 1.pdf available at https://authorea.com/users/353510/articles/495949-the-effect-oftreatment-on-cytokine-levels-in-non-muscle-invasive-bladder-cancer

\section{Hosted file}

Figure 2.pdf available at https://authorea.com/users/353510/articles/495949-the-effect-oftreatment-on-cytokine-levels-in-non-muscle-invasive-bladder-cancer

\section{Hosted file}

Figure 3.pdf available at https://authorea.com/users/353510/articles/495949-the-effect-oftreatment-on-cytokine-levels-in-non-muscle-invasive-bladder-cancer 